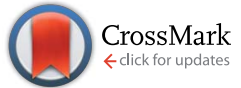

Cite this: RSC Adv., 2017, 7, 3594

Received 4th November 2016 Accepted 4th December 2016

DOI: $10.1039 / c 6 r a 26332 a$

www.rsc.org/advances

\section{Microwave-assisted synthesis of a viologen-based covalent organic polymer with redox-tunable polarity for dye adsorption $\uparrow$}

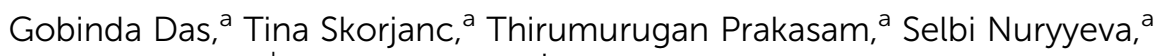 \\ John-Carl Olsen ${ }^{\mathrm{b}}$ and Ali Trabolsi ${ }^{\star a}$
}

\begin{abstract}
We report the efficient synthesis of a viologen-based covalent organic polymer by a microwave-assisted Menshutkin reaction. The polymer's viologen sub-units were synthesized in the 4,4'-bipyridinium dicationic state but could be reduced to radical-cationic and neutral forms. Regardless of the redox state of its viologens, the polymer was found to be thermally stable and capable of removing three dyes of differing polarities-fluorescein ( $F L)$, rhodamine $B(R h B)$, and Nile red (NR)-from aqueous solutions. With its viologens in the dicationic state, the polymer was most efficient at removing $\mathrm{FL}$, which is hydrophilic. With its viologens in the neutral state, it was most efficient at removing NR, which is hydrophobic. This strategy of using a single redox-tunable material for adsorbing multiple contaminants of different polarities represents a unique approach to water purification.
\end{abstract}

Water contamination caused by organic dyes from industrial waste is a major threat to the environment. ${ }^{1}$ Consequently, a considerable research effort has been undertaken to develop methods for capturing organic dyes. ${ }^{2}$ A wide variety of materials, including porous metal organic frameworks (MOFs) ${ }^{3-5}$ covalent organic frameworks (COFs), ${ }^{\mathbf{1 , 6}, 7}$ carbon-based materials, ${ }^{8-11}$ nanocomposites, ${ }^{\mathbf{1 2}, 13}$ and polymers, ${ }^{\mathbf{1 4 - 1 6}}$ has been fabricated to facilitate their removal from waste waters. In order to have an effective material for dye-removal, the polarity of the material must be similar to that of the adsorbate molecules. It is therefore challenging to design a single material for the removal of different organic dyes because different dyes can have polarities that range from hydrophobic to hydrophilic. Many strategies have been used to adjust a host material's surface polarity. ${ }^{17-19}$ However, these methods produce adsorbents with invariant polarities that are not usually effective for adsorbing both hydrophilic and hydrophobic dyes. Furthermore, developing and scaling-up the syntheses of multiple adsorbents is not cost effective.

Viologens are a class of compounds that contain the $4,4^{\prime}$ bipyridinium unit and are well known for their rich redox chemistry. Viologens have three redox states that can be accessed chemically or electrochemically and can range from hydrophilic to hydrophobic. ${ }^{20}$ Viologen-based materials are therefore strong candidates for the adsorption of multiple dyes that have different polarities. ${ }^{21}$

${ }^{a}$ New York University Abu Dhabi, Saadiyat Island, P.O. Box 129188, Abu Dhabi, United Arab Emirates. E-mail: ali.trabolsi@nyu.edu

${ }^{b}$ School of Sciences, Indiana University Kokomo, Kokomo, IN 46904, USA

$\dagger$ Electronic supplementary information (ESI) available. See DOI: 10.1039/c6ra26332a
Herein, we report the synthesis, via microwave-assisted Menshutkin reaction, of a cationic covalent organic polymer, $\mathbf{C O P}^{++}$, in which bipyridinium units crosslink hexatopic cyclotriphosphazene (CTP) core moieties (Fig. 1). ${ }^{22,23}$

Although the prepared polymer was found to be non-porous, its cationic nature and rich redox chemistry prompted us to

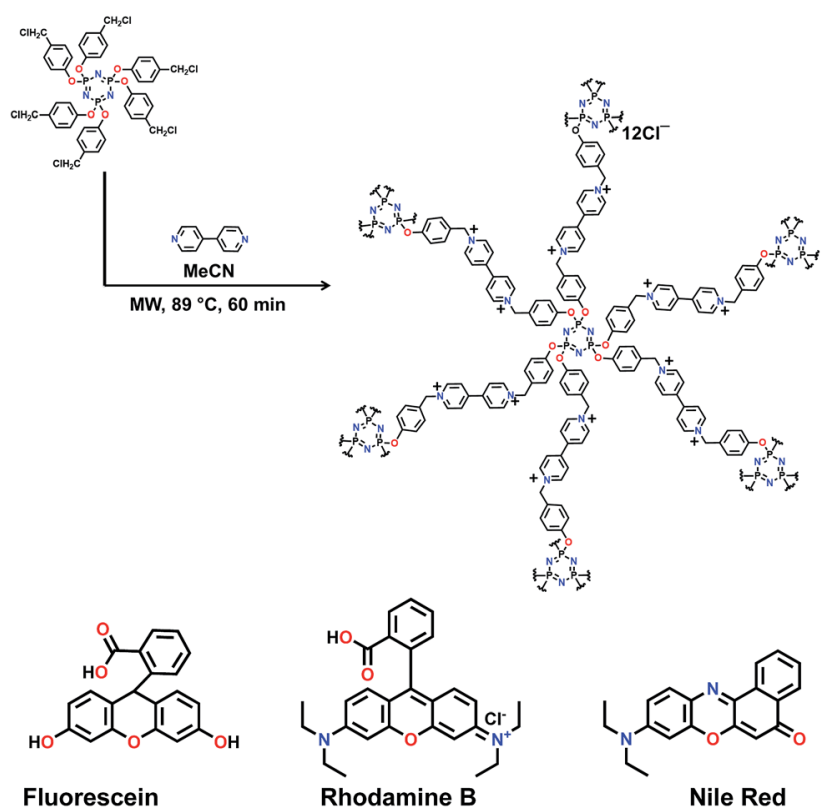

Fig. 1 Top: Microwave-assisted Menshutkin reaction to form $\mathrm{COP}_{1}{ }^{++}$. Bottom: Chemical structures of the three organic dyes used in this study. 
study its interactions, in aqueous solutions, with three dyes of different polarities - fluorescein (FL), rhodamine B (RhB), and Nile red (NR). FL is hydrophilic and formally neutral, RhB is hydrophilic with a positively charged center, and NR is a hydrophobic dye.

The polarity of the polymer could be adjusted from hydrophilic, in its dicationic and radical-cationic forms $\left(\mathbf{C O P}^{++}\right.$and $\mathbf{C O P}_{\mathbf{1}}{ }^{+}$, respectively) to hydrophobic, in its neutral form $\left(\mathbf{C O P}^{\mathbf{0}}\right)$, to match dye polarity. We accessed radical cationic and neutral forms by reaction with sodium dithionite and cobaltocene, respectively, and measured the adsorption of each dye to the polymer in each of its redox states. Consistent with its cationic nature, $\mathbf{C O P}_{\mathbf{1}}{ }^{++}$exhibited fast and selective adsorption $(100 \%)$ of electron-rich FL. Its adsorption of NR, on the other hand, was relatively slow and occurred to a much lesser extent (25\%). In contrast, the neutral form of the polymer, $\mathbf{C O P}_{\mathbf{1}}{ }^{\mathbf{0}}$ adsorbed NR quickly and to a significant extent (85\%). The radical-cationic polymer showed moderate to low adsorption efficiency for all three dyes.

To synthesize $\mathbf{C O P}_{\mathbf{1}}{ }^{++}$, a solution of 4,4'-dipyridyl (0.54 g, 3.5 $\mathrm{mmol})$ in $\mathrm{MeCN}(50 \mathrm{~mL})$ was added to hexakis(4chloromethylphenoxy)cyclotriphosphazene (1.0 g, $1.02 \mathrm{mmol})$ in MeCN $(50 \mathrm{~mL})$. The reaction mixture was stirred under microwave irradiation of $2.45 \mathrm{GHz}$ at $89{ }^{\circ} \mathrm{C}$ for $1 \mathrm{~h}$ and subsequently cooled to room temperature (Fig. 1 and Scheme S1 $\dagger$ ). The precipitate was filtered, washed with $\mathrm{MeCN}, \mathrm{H}_{2} \mathrm{O}$, acetone and $n$-hexane and dried under vacuum for $72 \mathrm{~h}$ to afford $\mathbf{C O P}_{\mathbf{1}}{ }^{++}$ as a yellow powder $(1.56 \mathrm{~g})$. The successful formation of the polymer was confirmed by Fourier transform infrared (FTIR) spectroscopy (Fig. S1 $\dagger$ ) and solid state cross polarization magic angle spinning (CP/MAS) ${ }^{13} \mathrm{C}$ NMR (Fig. S2 $\dagger$ ). In the FTIR spectrum of $\mathbf{C O P}_{\mathbf{1}}{ }^{++}$, the absence of an absorption characteristic of aliphatic $\mathrm{C}-\mathrm{Cl}$ stretching at $650-750 \mathrm{~cm}^{-1}$, and the presence of a strong absorption due to $\mathrm{CH}_{2}$ wagging at $1155 \mathrm{~cm}^{-1}$, confirmed the successful polymerization of hexakis(4-chloromethylphenoxy) cyclotriphosphazene and $4-4^{\prime}$ bipyridyl. The solid state CP/MAS ${ }^{13} \mathrm{C}$ NMR spectra shows (Fig. S2 $\dagger$ ) a prominent peak near $65 \mathrm{ppm}$, which corresponds to the $-\mathrm{NCH}_{2}$ carbon and further confirmed polymer formation. $\mathbf{C O P}_{\mathbf{1}}{ }^{++}$was not soluble in common organic solvents and was thermally more stable than the corresponding monomers (Fig. S4-S6†). Upon thermogravimetric analysis (TGA), $\mathbf{C O P}_{\mathbf{1}}{ }^{++}$exhibited an initial weight loss of $5-10 \%$ below $200{ }^{\circ} \mathrm{C}$, mainly due to solvent evaporation, and a steep weight loss at about $212{ }^{\circ} \mathrm{C}$ (Fig. S4$\mathrm{S} 6 \dagger^{\dagger}$ ). The broad pattern in its powder X-ray diffraction spectrum (Fig. $\mathrm{S} 7 \dagger)$ and the roughly spherical $(1 \mu \mathrm{m}$ average diameter) particles visible in scanning electron spectroscopy (SEM) images (Fig. 2) indicate that $\mathbf{C O P}_{\mathbf{1}}{ }^{++}$is amorphous. The absence of signal corresponding to nitrogen gas uptake in $\mathrm{N}_{2}$ adsorption isotherms (Fig. S8a $\dagger$ ) measured at $77 \mathrm{~K}$ reveals that it is nonporous. The non-porous character of the synthesized polymer was further confirmed by measurement of its inability to adsorb $\mathrm{CO}_{2}$ (Fig. S8b $\dagger$ ) at different temperatures.

Sodium dithionite $\left(\mathrm{Na}_{2} \mathrm{~S}_{2} \mathrm{O}_{4}\right)$ and cobaltocene were used to reduce $\mathbf{C O P}_{\mathbf{1}}{ }^{++}$in an oxygen-free environment to afford the radical-cationic $\mathbf{C O P}_{\mathbf{1}}{ }^{+}$, and the neutral $\mathbf{C O P}_{\mathbf{1}}{ }^{\mathbf{}}$ forms, respectively. $\mathbf{C O P}_{1}{ }^{++}(30 \mathrm{mg})$ and an excess of $\mathrm{Na}_{2} \mathrm{~S}_{2} \mathrm{O}_{4}$ or

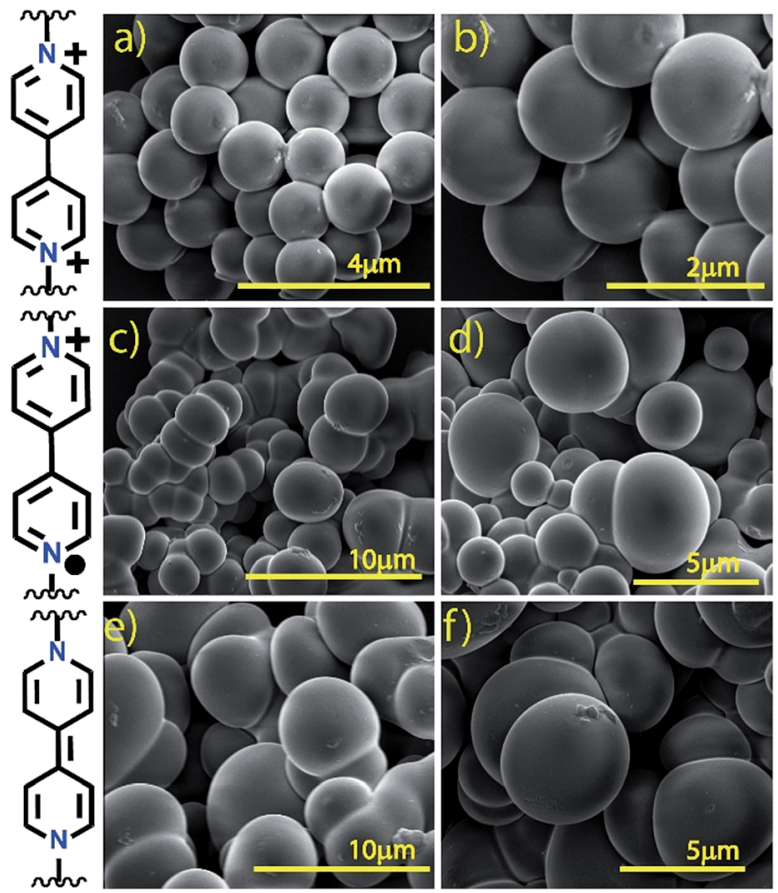

Fig. 2 Scanning electron microscopy images of the viologen-linked covalent organic polymer in different redox states: $\mathrm{COP}_{1}{ }^{++}$cationic

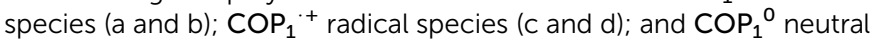
species (e and f). The cationic form exhibits single-sphere morphology, while the radical and the neutral polymers are more aggregated.

cobaltocene were added to degassed $\mathrm{H}_{2} \mathrm{O}$ or $\mathrm{CH}_{3} \mathrm{CN}$ under a nitrogen atmosphere, and the mixture was shaken for one minute. Upon addition of $\mathrm{Na}_{2} \mathrm{~S}_{2} \mathrm{O}_{4}$ or cobaltocene, the color of the solid powder instantly changed from yellow to dark blue or brown, respectively. With the aid of centrifugation $(4000 \mathrm{rpm}$, $15 \mathrm{rpm}$ ), the solid was then filtered and washed three times with degassed $\mathrm{H}_{2} \mathrm{O}$ under $\mathrm{N}_{2}$ atmosphere. The solid-state EPR spectrum of the powder recovered after reduction with $\mathrm{Na}_{2} \mathrm{~S}_{2} \mathrm{O}_{4}$ reveals (Fig. S9†) a sharp, well-defined peak that reflects the presence of unpaired electrons and therefore the formation of the radical-cationic form of the polymer, $\mathbf{C O P}_{\mathbf{1}}{ }^{+}$. The solid state UV-Vis spectrum of $\mathbf{C O P}_{\mathbf{1}}{ }^{+}$displays (Fig. S10†) a broad band at $\sim 605 \mathrm{~nm}$, that is also indicative of radicals. The lack of signal in the EPR spectrum of the powder isolated after reduction with cobaltocene (Fig. S9c $\dagger$ ) indicates formation of the neutral, diamagnetic form of the polymer. SEM images of the reduced forms of the polymer confirm their structural integrity, as no changes in the size of their particles and only slight changes in the morphology of their particles are apparent (Fig. 2c-f). TGA analysis show that all three forms, $\mathbf{C O P}_{\mathbf{1}}{ }^{++}, \mathbf{C O P}_{\mathbf{1}}{ }^{++}$and $\mathbf{C O P}_{\mathbf{1}}{ }^{\mathbf{}}$, are thermally stable up to $400{ }^{\circ} \mathrm{C}$ (Fig. S2-S4†). Remarkably, $\mathbf{C O P}_{\mathbf{1}}{ }^{+}$was found to be stable for 10-15 days under ambient conditions. The enhanced stability of the radical cationic state within the polymer, relative to its more fleeting existence within the monomer, can be explained by steric protection as well as radical-radical and other stabilizing interactions that can occur within the polymeric structure. 
FTIR spectra of the reduced forms of the polymer reveal (Fig. S1 $\dagger$ ) pronounced intensity changes compared to the FTIR spectrum of $\mathbf{C O P}_{\mathbf{1}}{ }^{++}$. Such changes may be the result of the electron transfer and reflect significant perturbations of bond polarities. ${ }^{24}$ In contrast, the frequencies of most absorptions are not drastically different than those of the corresponding absorptions in the spectrum of $\mathbf{C O P}_{\mathbf{1}}{ }^{++}$. Thus, the structural deformations that are caused by reduction are probably largely delocalized over many bonds.

The thermal and hydrolytic stability of our material and our having the means to effectively control its polarity by chemical reduction prompted us to exam its ability to adsorb and remove three organic dyes having different polarities from aqueous solutions. Of the dyes used, FL and RhB are hydrophilic and NR is hydrophobic. In addition, RhB is a positively charged molecule with a $\mathrm{Cl}^{-}$counter anion. About $30 \mathrm{mg}$ of $\mathbf{C O P}_{\mathbf{1}}{ }^{++}$was added to $10 \mathrm{~mL}$ of each of the following dye solutions: $1 \times 10^{-4} \mathrm{M}$ aqueous FL, $1 \times 10^{-4} \mathrm{M}$ aqueous RhB and $2 \times 10^{-4} \mathrm{M}$ NR in a $1: 1$ water: methanol mixture (Fig. 3). Absorbance was monitored by UV-Vis spectrophotometry prior to and at 10 minutes intervals after polymer addition. Bold orange lines in Fig. 3 correspond to the initial and final absorbance measurements of each dye solution. The data indicate that FL is readily adsorbed by both $\mathbf{C O P}_{\mathbf{1}}{ }^{++}$and $\mathbf{C O P}_{\mathbf{1}}{ }^{++}$(Fig. 3a and b). Strong hydrophilic-hydrophilic interactions between $\mathrm{FL}$ and $\mathbf{C O P}_{\mathbf{1}}{ }^{++}$ result in a complete disappearance of the dye's absorption peak. COP $_{1}{ }^{++}$removed $\sim 99 \%$ of the FL in only 4 min (Fig. 4 ), which represents one of the fastest adsorption rates reported for organic polymers. ${ }^{25} \mathbf{C O P}_{\mathbf{1}}{ }^{+}$and $\mathbf{C O P}_{\mathbf{1}}{ }^{\mathbf{}}$ removed $\sim 40 \%$ and $\sim 11 \%$ of the FL, respectively, after 60 minutes (Fig. 4), which indicates that FL has less affinity for the more hydrophobic, reduced forms of the polymer.

$\mathbf{C O P}_{\mathbf{1}}{ }^{++}$removed the hydrophilic RhB less efficiently than FL. A slow but steady decrease of RhB's absorption intensity in the UV-Vis measurements indicated $\sim 25 \%$ removal after one hour (Fig. 3d). This lower efficiency may be explained by the electrostatic repulsion between the positively charged polymer and the positively charged dye. $\mathbf{C O P}_{\mathbf{1}}{ }^{++}$was slightly less efficient at removing RhB than $\mathbf{C O P}_{1}{ }^{++}$, adsorbing $\sim 20 \%$ of the dye after one hour (Fig. 3e and 4). Consistent with its increasing hydrophobic character, $\mathbf{C O P}_{\mathbf{1}}{ }^{\mathbf{0}}$ was the least efficient, removing a barely detectible amount of RhB after an hour (Fig. 3f and 4).

$\mathbf{C O P}_{1}{ }^{++}$removed a moderate amount $(25 \%)$ of NR (Fig. $3 \mathrm{~g}$ and 4) after one hour, reflecting a weak interaction between this hydrophobic dye and the positively charged polymer. $\mathbf{C O P}_{\mathbf{1}}{ }^{+}$ removed slightly less NR (Fig. $3 \mathrm{~h}$ ). In contrast, $\mathbf{C O P}_{\mathbf{1}}{ }^{\mathbf{}}$ removed $85 \%$ of the NR within one hour, as a result of increased hydrophobic interactions (Fig. 3i and 4).
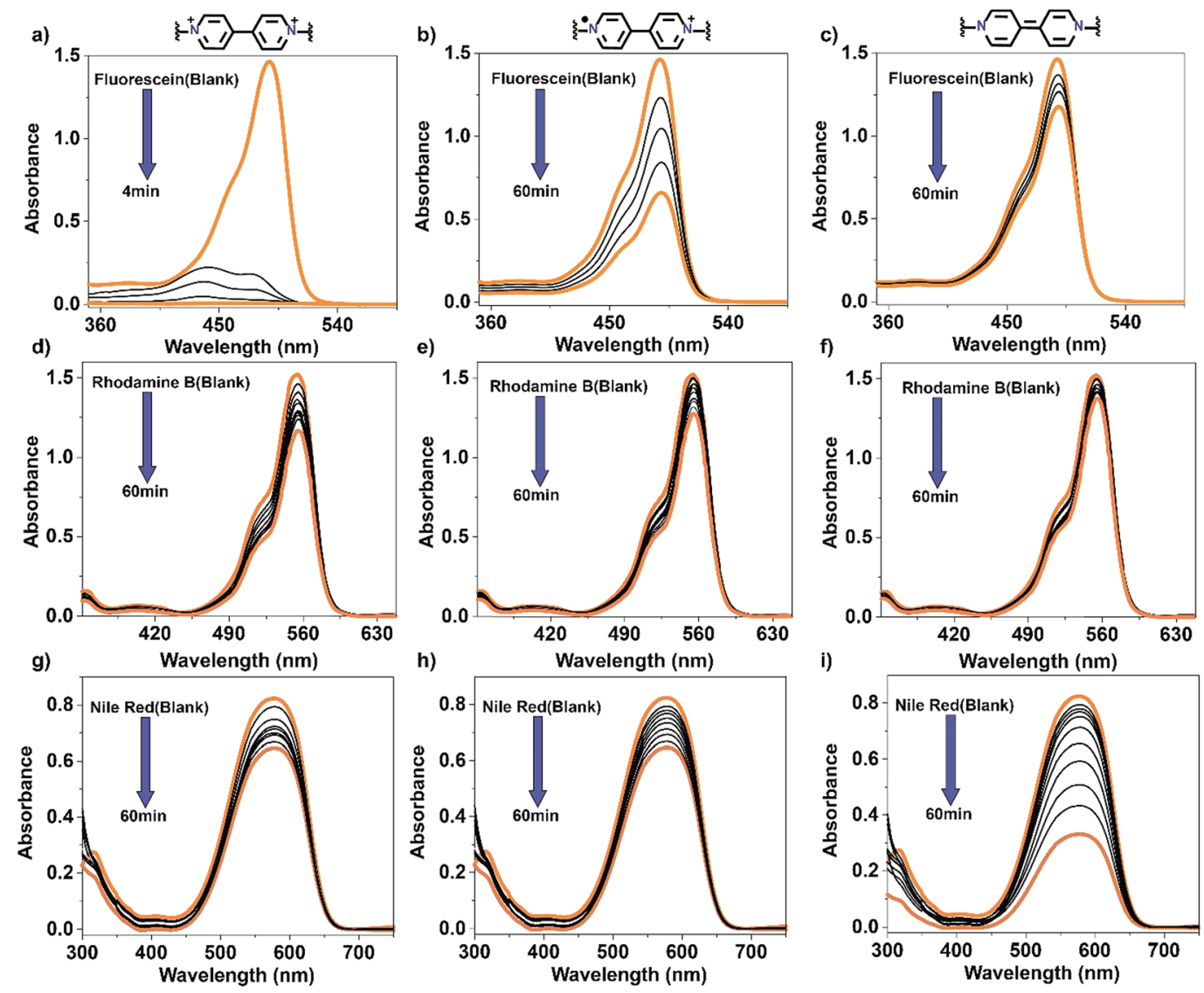

Fig. 3 Spectroscopic monitoring of dye removal. (a-c) UV/Vis spectra of aqueous solutions of fluorescein and $\mathrm{COP}_{1}{ }^{++}(\mathrm{a}), \mathrm{COP}_{1}{ }^{+}(\mathrm{b}), \mathrm{or}^{-}$ $\mathrm{COP}_{1}{ }^{0}$ (c). (d-f) UV/Vis spectra of aqueous solutions of rhodamine $\mathrm{B}$ and $\mathrm{COP}_{1}{ }^{++}$(d), $\mathrm{COP}_{1}{ }^{+}(\mathrm{e})$, and $\mathrm{COP}_{1}{ }^{0}(\mathrm{f})$. (g-i) UV/Vis spectra of $1: 1$ water : methanol solutions of Nile red and $\mathrm{COP}_{1}^{++}(\mathrm{g}), \mathrm{COP}_{1}{ }^{+}(\mathrm{h})$, and $\mathrm{COP}_{1}{ }^{0}$ (i). 


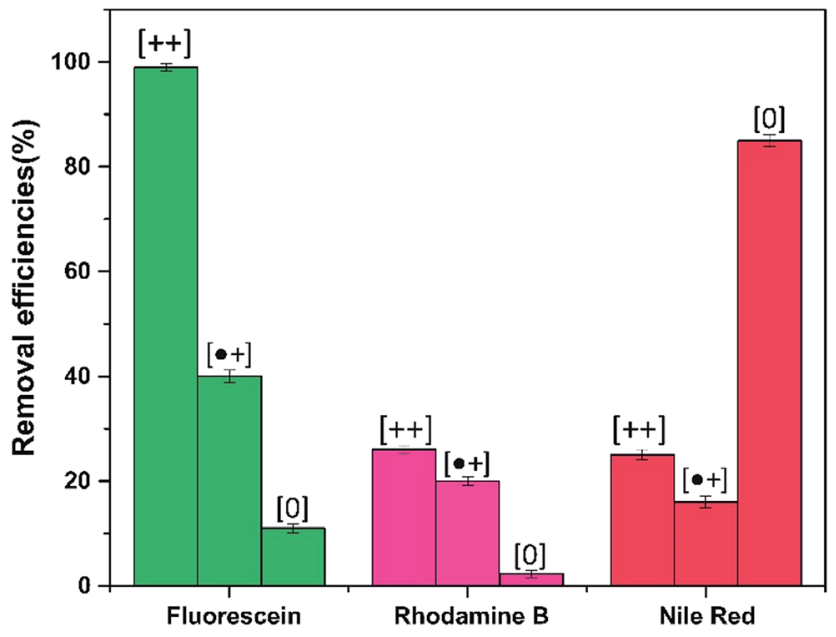

Fig. 4 Adsorption capacities of the cationic, radical-cationic and neutral forms of $\mathrm{COP}_{1}$ for fluorescein, rhodamine B, and Nile Red. Adsorption time was one hour in all cases.

In order to recycle the different forms of the polymer, we conducted regeneration tests that corresponded to the cases where dye removal had been most efficient: for $\mathbf{C O P}_{\mathbf{1}}{ }^{++}$removal of FL and for $\mathbf{C O P}_{\mathbf{1}}{ }^{\mathbf{0}}$ removal of NR. In these experiments, dyeloaded polymer samples were dried at $60{ }^{\circ} \mathrm{C}$ and then immersed in different solvents at room temperature. Dye release was monitored by UV-Vis and fluorescence spectroscopies. The release of FL from FL-loaded $\mathbf{C O P}_{\mathbf{1}}{ }^{++}$was measured in a saturated aqueous solution of sodium chloride and confirmed by an increase in the intensity of FL's characteristic absorption band. Ninety percent of the FL was released within one hour (Fig. S11 $\dagger$ ). We infer that the competitive binding of chloride anions to the polymer facilitates the release of FL molecules.

NR was removed from $\mathbf{C O P}_{\mathbf{1}}{ }^{\mathbf{0}}$ by immersion in methanol, and the process was followed by fluorescence spectroscopy. The neutral polymer released $\sim 90 \%$ of the dye in one hour (Fig. S12 and $\mathrm{S} 13 \dagger)$. After regeneration, the polymer samples were analyzed by FTIR (Fig. S14 $\dagger$ ) and SEM (Fig. S15 $\dagger$ ), and their spherical morphologies were found to be intact, confirming their structural robustness.

In summary, microwave radiation was used to efficiently prepare, by Menshutkin coupling, a thermally stable cationic polymer, in which 4,4'-bipyridinium units link hexatopic phosphazene core moieties. The redox responsive viologen units of the polymer allowed access to radical cationic and neutral states. Switching the viologens' redox states allowed the polymer's polarity to be adjusted from the hydrophilic dicationic form to hydrophobic neutral one. The three forms of the polymer were tested for their ability to remove three organic dyes from aqueous solutions. The cationic polymer was highly selective and efficient for the adsorption of fluorescein, which is a hydrophilic dye; showed moderate uptake of rhodamine $\mathrm{B}$, which is positively charged and hydrophilic; and demonstrated poor uptake for Nile red, which is hydrophobic. The radical cationic form of the polymer displayed poor uptake and selectivity for all three dyes. The neutral form was highly selective for Nile red as compared to fluorescein and rhodamine $\mathrm{B}$. Thus, this versatile polymer with its easily adjustable redox states is a promising material for the removal of dyes of different polarities from waste water.

\section{Acknowledgements}

The research described here was sponsored by New York University Abu Dhabi (NYUAD), UAE. G. D., T. S., T. P., S. N. and A. T. thank NYUAD for its generous support of the research program at NYUAD. The research was carried out by using the Core Technology Platform resources at NYUAD. The authors thank Professor Kyriaki Polychronopoulou for the $\mathrm{CO}_{2}$ gas uptake measurements.

\section{Notes and references}

1 S.-B. Yu, H. Lyu, J. Tian, H. Wang, D.-W. Zhang, Y. Liu and Z.-T. Li, Polym. Chem., 2016, 7, 3392-3397.

2 A. K. Gupta, R. R. Naregalkar, V. D. Vaidya and M. Gupta, Nanomedicine, 2007, 2, 23-39.

3 X. Zhao, S. Liu, Z. Tang, H. Niu, Y. Cai, W. Meng, F. Wu and J. P. Giesy, Sci. Rep., 2015, 5, 11849.

4 S. Duan, J.-X. Li, X. Liu, Y. Wang, S. Zeng, D. Shao and T. Hayat, ACS Sustainable Chem. Eng., 2016, 4, 3368-3378.

5 Y.-C. He, J. Yang, W.-Q. Kan, H.-M. Zhang, Y.-Y. Liu and J.-F. Ma, J. Mater. Chem. A, 2015, 3, 1675-1681.

6 W. Zhang, F. Liang, C. Li, L.-G. Qiu, Y.-P. Yuan, F.-M. Peng, X. Jiang, A.-J. Xie, Y.-H. Shen and J.-F. Zhu, J. Hazard. Mater., 2011, 186, 984-990.

7 T. Wang, K. Kailasam, P. Xiao, G. Chen, L. Chen, L. Wang, J. Li and J. Zhu, Microporous Mesoporous Mater., 2014, 187, 63-70.

8 Z. Zhang and J. Kong, J. Hazard. Mater., 2011, 193, 325-329. 9 J. N. Tiwari, K. Mahesh, N. H. Le, K. C. Kemp, R. Timilsina, R. N. Tiwari and K. S. Kim, Carbon, 2013, 56, 173-182.

10 B. Chen, Q. Ma, C. Tan, T. Lim, L. Huang and H. Zhang, Small, 2015, 11, 3319-3336.

11 X. Liu, L. Yan, W. Yin, L. Zhou, G. Tian, J. Shi, Z. Yang, D. Xiao, Z. Gu and Y. Zhao, J. Mater. Chem. A, 2014, 2, 12296-12303.

12 P. Hu, L. Han and S. Dong, ACS Appl. Mater. Interfaces, 2013, 6, 500-506.

13 A. K. Sarkar, A. Saha, A. Tarafder, A. B. Panda and S. Pal, ACS Sustainable Chem. Eng., 2016, 4, 1679-1688.

14 P. Kuhn, K. Krüger, A. Thomas and M. Antonietti, Chem. Commun., 2008, 5815-5817.

15 Z. Wang, J. Guo, J. Ma and L. Shao, J. Mater. Chem. A, 2015, 3, 19960-19968.

16 A. Alsbaiee, B. J. Smith, L. Xiao, Y. Ling, D. E. Helbling and W. R. Dichtel, Nature, 2015, 529, 190-194.

17 Z. Gan, A. Zhao, M. Zhang, W. Tao, H. Guo, Q. Gao, R. Mao and E. Liu, Dalton Trans., 2013, 42, 8597-8605.

18 T. Zhu, J. S. Chen and X. W. Lou, J. Phys. Chem. C, 2012, 116, 6873-6878. 
19 L. Xu, Y. Luo, L. Sun, S. Pu, M. Fang, R.-X. Yuan and H.-B. Du, Dalton Trans., 2016, 45, 8614-8621.

20 C. Hua, B. Chan, A. Rawal, F. Tuna, D. Collison, J. M. Hook and D. M. D'Alessandro, J. Mater. Chem. C, 2016, 4, 25352544.

21 G.-Q. Gao and A.-W. Xu, New J. Chem., 2014, 38, 4661-4665.

22 K. Wadhwa, S. Nuryyeva, A. C. Fahrenbach, M. Elhabiri, C. P. Iglesias and A. Trabolsi, J. Mat. Chemi. C, 2013, 1, 2302-2307.
23 G. Das, T. Prakasam, S. Nuryyeva, D. Suk Han, A. AbdelWahab, J.-C. Olsen, K. Polychronopoulou, C. PlatasIglesias, M. Jouiad, F. Ravaux and A. Trabolsi, J. Mater. Chem. A, 2016, 4, 15361-15369.

24 O. Poizat, C. Sourisseau and J. Corset, J. Mol. Struct., 1986, 143, 203-206.

25 S. Pirillo, L. Cornaglia, M. L. Ferreira and E. H. Rueda, Spectrochim. Acta, Part A, 2008, 71, 636-643. 